\title{
Construction of MDS Self-Dual Codes over Galois Rings
}

\author{
${ }^{*}$ Jon-Lark $\mathrm{Kim}^{a}$ \\ Department of Mathematics \\ University of Louisville \\ Louisville, KY 40292, USA \\ E-mail: jl.kim@louisville.edu \\ Yoonjin Lee ${ }^{b}$ \\ Department of Mathematics \\ Simon Fraser University \\ Burnaby, BC, v5a 1s6, Canada \\ E-mail: yoonjinl@sfu.ca
}

July 17, 2007

\begin{abstract}
The purpose of this paper is to construct nontrivial MDS self-dual codes over Galois rings. We consider a building-up construction of self-dual codes over Galois rings as a $\operatorname{GF}(q)$-analogue of [20]. We give a necessary and sufficient condition on which the building-up construction holds. We construct MDS self-dual codes of lengths up to 8 over $\operatorname{GR}\left(3^{2}, 2\right), \operatorname{GR}\left(3^{3}, 2\right)$ and $\operatorname{GR}\left(3^{4}, 2\right)$, and near-MDS self-dual codes of length 10 over these rings. In a similar manner, over $\operatorname{GR}\left(5^{2}, 2\right), \operatorname{GR}\left(5^{3}, 2\right)$ and $\operatorname{GR}\left(7^{2}, 2\right)$, we construct MDS self-dual codes of lengths up to 10 and near-MDS self-dual codes of length 12. Furthermore, over $\operatorname{GR}\left(11^{2}, 2\right)$ we have MDS self-dual codes of lengths up to 12 .
\end{abstract}

Key words. Self-dual code, Galois ring, MDS code.

\section{Introduction}

Codes over $\mathbb{Z}_{4}$ have intrigued a lot of researchers thanks to the discovery that Kerdock and Preparata codes are linear over $\mathbb{Z}_{4}$ via the Gray map from $\mathbb{Z}_{4}^{n}$ to $\mathbb{Z}_{2}^{2 n}$, and that they are

${ }^{*}$ The author ${ }^{a}$ was supported in part by a Project Completion Grant from the University of Louisville and the author ${ }^{b}$ was supported by NSERC. 
duals as $\mathbb{Z}_{4}$-codes [18]. It is worth noting that even before this remarkable result there had have been interesting work on codes over $\mathbb{Z}_{m}$ ([2], [3], [30], [31]), and codes over Galois rings $\operatorname{GR}\left(p^{e}, l\right)$ (e.g., [29]). Recently codes over finite chain rings (e.g., [26] and references therein) and codes over finite Frobenius rings (e.g., [33]) have been studied.

One of the interesting classes of codes over finite rings is the class of self-dual codes over these rings. Self-dual codes over $\mathbb{Z}_{4}$ (e.g., [4]) have been generalized to self-dual codes over $\mathbb{Z}_{2 k}$ with connections to even unimodular lattices and modular forms [1], to self-dual codes over $\mathbb{Z}_{8}$ and $\mathbb{Z}_{9}[12]$, to self-dual codes over $\mathbb{Z}_{m}[11]$, and to self-dual codes over finite principal ideal rings [14]. We refer to the chapter on self-dual codes in the Handbook of Coding Theory [28] for a detailed description. Recently, self-dual codes over Galois rings have been added as one of the important types of self-dual codes with their connection to invariant theory [24]. Finding interesting (nontrivial) examples of self-dual codes over Galois rings $\operatorname{GR}\left(p^{r}, m\right)$ is proposed as one of the research problems in [24, Sec. 2.4.10]. This motivates our current study, i.e., finding good self-dual codes over general Galois rings.

We notice that $\operatorname{GR}(p, r)=\operatorname{GF}\left(p^{r}\right)$ (the finite field of order $p^{r}$ ) and $\operatorname{GR}\left(p^{m}, 1\right)=\mathbb{Z}_{p^{m}}$. In $[17,20]$, constructions of self-dual codes over $\operatorname{GF}\left(p^{r}\right)$ have been developed, and self-dual codes over $\mathbb{Z}_{p^{m}}$ have been studied in many places, for instance, [6, 8, 10, 13, 21, 27]. Gaborit, et. al. [16] have constructed an infinite family of self-dual quadratic double circulant codes over GR $(4,2)$. MDS self-dual codes over $\operatorname{GR}\left(2^{m}, r\right)$ have been constructed using ReedSolomon codes [14]. Little is known about MDS self-dual codes over $\operatorname{GR}\left(p^{m}, r\right)$ when $p$ is odd and $r>1$.

In this paper, we suggest a general method called a building-up construction of self-dual codes over Galois rings as a GF( $q$ )-analogue of [20]. We give a necessary and sufficient condition on which the building-up construction holds. Our method is very efficient in searching for self-dual codes over Galois rings. We generate examples of self-dual codes over a Galois ring $\operatorname{GR}\left(p^{m}, r\right)$ when $m=2,3$, or 4 , and $r=2$. More precisely, we give MDS self-dual codes for lengths up to 8 over $\operatorname{GR}\left(3^{2}, 2\right), \operatorname{GR}\left(3^{3}, 2\right)$ and $\operatorname{GR}\left(3^{4}, 2\right)$, and near-MDS self-dual codes of length 10 over these rings. In a similar manner, over $\operatorname{GR}\left(5^{2}, 2\right), \operatorname{GR}\left(5^{3}, 2\right)$ and $\operatorname{GR}\left(7^{2}, 2\right)$, we construct MDS self-dual codes of lengths up to 10 and near-MDS selfdual codes of length 12. Furthermore, over GR $\left(11^{2}, 2\right)$ we have MDS self-dual codes of lengths up to 12. Our results on the existence of MDS self-dual codes over $\operatorname{GR}\left(p^{m}, 2\right)$ are summarized in Table 1. All examples in this paper are constructed using Magma [7].

\section{Preliminaries}

We give a brief introduction to some basic definitions and facts regarding linear codes [6], [26] over Galois rings [22], [32].

Let $p$ be a fixed prime and $m$ a positive integer. Let $f$ be a polynomial in $\mathbb{Z}_{p^{m}}[x]$ and $\bar{f}$ the image of $f$ under the projection $\mathbb{Z}_{p^{m}}[x] \rightarrow \mathbb{Z}_{p}[x]$. Then $f$ is called basic irreducible 
Table 1: Summary on the existence of MDS self-dual codes of length $n$ over $\operatorname{GR}\left(p^{m}, 2\right)$

\begin{tabular}{c|c|l}
\hline$p$ & $m$ & length $n$ \\
\hline \multirow{3}{*}{3} & 2 & $2,4,6,8$ \\
& 3 & $2,4,6,8$ \\
& 4 & $2,4,6,8$ \\
\hline 5 & 2 & $2,4,6,8,10$ \\
& 3 & $2,4,6,8,10$ \\
\hline 7 & 2 & $2,4,6,8,10$ \\
\hline 11 & 2 & $2,4,6,8,10,12$ \\
\hline
\end{tabular}

if both $f$ and $\bar{f}$ are irreducible. Let $r$ be the degree of $f$. A Galois ring is constructed as $\operatorname{GR}\left(p^{m}, r\right)=\mathbb{Z}_{p^{m}}[x] /(f)$, where $f$ is a monic basic irreducible polynomial in $\mathbb{Z}_{p^{m}}[x]$ of degree $r$. We note that $\operatorname{GR}\left(p^{m}, r\right)$ has $p^{m r}$ elements. We observe that $\operatorname{GR}(p, r)=\operatorname{GF}\left(p^{r}\right)$ (the finite field of order $p^{r}$ ) and $\operatorname{GR}\left(p^{m}, 1\right)=\mathbb{Z}_{p^{m}}$.

A linear code $\mathcal{C}$ of length $n$ over $\operatorname{GR}\left(p^{m}, r\right)$ is a submodule of $\operatorname{GR}\left(p^{m}, r\right)^{n}$. Let $\operatorname{GR}\left(p^{m}, r\right)^{*}$ be the unit group of $\operatorname{GR}\left(p^{m}, r\right)$. Then $\operatorname{GR}\left(p^{m}, r\right)^{*}$ has the following group structure [22]: If $p$ is odd, or if $p=2$ and $m \leq 2$, then

$$
\operatorname{GR}\left(p^{m}, r\right)^{*} \simeq \mathbb{Z}_{p^{r}-1} \times \mathbb{Z}_{p^{m-1}} \times \mathbb{Z}_{p^{m-1}} \cdots \times \mathbb{Z}_{p^{m-1}}
$$

with $r$ copies of $\mathbb{Z}_{p^{m-1}}$.

If $p=2$ and $m \geq 3$, then

$$
\operatorname{GR}\left(2^{m}, r\right)^{*} \simeq \mathbb{Z}_{2^{r}-1} \times \mathbb{Z}_{2} \times \mathbb{Z}_{2^{m-2}} \times \mathbb{Z}_{2^{m-1}} \cdots \times \mathbb{Z}_{2^{m-1}},
$$

with $r-1$ copies of $\mathbb{Z}_{2^{m-1}}$.

A Galois ring $\operatorname{GR}\left(p^{m}, r\right)$ is a local ring with maximal ideal generated by $p$, and the nilpotency index of $p$ is $m$, that is, the smallest positive integer such that $p^{m}=0$ in $\operatorname{GR}\left(p^{m}, r\right)$. A generator matrix for a linear code $\mathcal{C}$ over $\operatorname{GR}\left(p^{m}, r\right)$ is permutation equivalent to the following one in the standard form [25], [26]:

$$
G=\left[\begin{array}{ccccccc}
I_{k_{0}} & A_{0,1} & A_{0,2} & A_{0,3} & \cdots & A_{0, m-1} & A_{0, m} \\
0 & p I_{k_{1}} & p A_{1,2} & p A_{1,3} & \cdots & p A_{1, m-1} & p A_{1, m} \\
0 & 0 & p^{2} I_{k_{2}} & p^{2} A_{2,3} & \cdots & p^{2} A_{2, m-1} & p^{2} A_{2, m} \\
\vdots & \vdots & \vdots & \vdots & & \vdots & \vdots \\
0 & 0 & 0 & 0 & \cdots & p^{m-1} I_{k_{m-1}} & p^{m-1} A_{m-1, m}
\end{array}\right]
$$

where the columns are grouped into square blocks of sizes $k_{0}, k_{1}, \cdots, k_{m-1}, k_{m}=n-$ $\sum_{i=0}^{m-1} k_{i}$ with $k_{i} \geq 0$ for $0 \leq i \leq m-1$. Then the code $\mathcal{C}$ over $\operatorname{GR}\left(p^{m}, r\right)$ is said to be of 
type

$$
(1)^{k_{0}}(p)^{k_{1}}\left(p^{2}\right)^{k_{2}} \cdots\left(p^{m-1}\right)^{k_{m-1}}
$$

with each $k_{i}$ a nonnegative integer. Given a code $C$ with this generator matrix, one has (see [26]) that

$$
|C|=p^{r\left(\sum_{i=0}^{m-1}(m-i) k_{i}\right)} .
$$

The rank of a code $\mathcal{C}$, denoted by $\operatorname{rank}(\mathcal{C})$, is defined to be the number of nonzero rows of its generator matrix $G$ in a standard form. Clearly $\operatorname{rank}(\mathcal{C})=\sum_{i=0}^{m-1} k_{i}$. We call $k_{0}$ in $G$ the free rank of a code $\mathcal{C}$.

The (Hamming) distance $d(\mathbf{x}, \mathbf{y})$ between two vectors $\mathbf{x}, \mathbf{y} \in \operatorname{GR}\left(p^{m}, r\right)^{n}$ is the number of coordinates in which $\mathbf{x}, \mathbf{y}$ differ. The (Hamming) weight of a codeword $\mathbf{c} \in \mathcal{C}$ is $d(\mathbf{c}, \mathbf{0})$. The (Hamming) minimum distance $d_{H}(\mathcal{C})$ of a code $\mathcal{C}$ is the smallest distance between distinct codewords. The minimum weight of all nonzero codewords in $\mathcal{C}$ is called the minimum weight of $\mathcal{C}$. Throughout this paper, an $\left[n, k, d_{H}\right]$ code denotes a linear code of length $n$ with $k=\operatorname{rank}(\mathcal{C})$ and the minimum distance (or minimum weight) $d_{H}$.

It is known (see [23] for example) that for (linear or nonlinear) codes $C$ of length $n$ over any finite alphabet $A$

$$
d_{H}(C) \leq n-\log _{|A|}(|C|)+1
$$

Codes meeting this bound are called MDS (Maximum Distance Separable) codes. These codes have been considered for their purely combinatorial properties. For example, they are related to the existence of mutually orthogonal Latin squares and orthogonal arrays (see [23, Ch. 11]).

Further if $C$ is linear over a ring, then

$$
d_{H}(C) \leq n-\operatorname{rank}(C)+1 .
$$

Codes meeting this bound are called MDR (Maximum Distance with respect to Rank) codes ([15], [26]).

The codes (if they are not free) meeting Equation (5) do not necessarily meet Equation (4). For example, the linear self-dual code over $\mathbb{Z}_{4}$ with generator matrix (2) is an MDR code but not an MDS code. It is easy to see that an MDR code which is free is necessarily an MDS code.

Under the usual inner product · on $\operatorname{GR}\left(p^{m}, r\right)^{n}$ the dual code $\mathcal{C}^{\perp}$ of $\mathcal{C}$ is defined as

$$
\mathcal{C}^{\perp}=\left\{\mathbf{x} \in \operatorname{GR}\left(p^{m}, r\right)^{n} \mid \mathbf{x} \cdot \mathbf{y}=0 \text { for all } \mathbf{y} \in \mathcal{C}\right\} .
$$

If $\mathcal{C} \subseteq \mathcal{C}^{\perp}$, then $\mathcal{C}$ is called a self-orthogonal code. We call $\mathcal{C}$ a self-dual code if $\mathcal{C}=\mathcal{C}^{\perp}$. Furthermore, the type of the dual code $\mathcal{C}^{\perp}$ of $\mathcal{C}$ can be determined as follows [25]:

$$
(1)^{k_{m}}(p)^{k_{m-1}}\left(p^{2}\right)^{k_{m-2}} \cdots\left(p^{m-1}\right)^{k_{1}} .
$$

It is known that $|\mathcal{C}|\left|\mathcal{C}^{\perp}\right|=p^{m r n}([26],[33])$. 


\section{Building-up construction for self-dual codes over Galois rings}

In this section, we present a building-up construction method for self-dual codes over $\operatorname{GR}\left(p^{m}, r\right)$; this is an extension of the building-up construction for self-dual codes over $G F\left(p^{m}\right)[20]$ and over $\mathbb{Z}_{p^{m}}$ [21] to linear codes over $\operatorname{GR}\left(p^{m}, r\right)$. We remark that similar building-up constructions were already treated for binary self-dual codes in [5] and for self-dual codes over finite fields in [9].

Our construction method requires the existence of $c$ in $\operatorname{GR}\left(p^{m}, r\right)$ such that $c^{2}=-1$ in $\operatorname{GR}\left(p^{m}, r\right)$. The following lemmas show the necessary and sufficient condition for -1 to be a square in $\operatorname{GR}\left(p^{m}, r\right)$.

Lemma 3.1. Let $p$ be an odd prime. Then -1 is a square in $\operatorname{GR}\left(p^{m}, r\right)$ if and only if either $p \equiv 1(\bmod 4)$ with any $r$ or $p \equiv-1(\bmod 4)$ with $r$ even.

Proof. Let $\operatorname{GR}\left(p^{m}, r\right)=\mathbb{Z}_{p^{m}}[x] /(f)$. We show that -1 is a square in $\operatorname{GR}\left(p^{m}, r\right)$ if and only if

$$
(-1)^{\frac{p^{r}-1}{2}}=1 \text { in } \mathbb{F}_{p^{r}}^{*}
$$

For simplicity, we denote $\operatorname{GR}\left(p^{m}, r\right)$ by $R$. The natural reduction $\bmod p$ map

$$
\psi: R^{*}=\left(\mathbb{Z}_{p^{m}}[x] /(f)\right)^{*} \rightarrow(R /(p))^{*}=\left(\mathbb{Z}_{p}[x] /(\bar{f})\right)^{*}
$$

is a surjective homomorphism whose image has order $p^{r}-1$, and its kernel is a $p$-group from Eq. (1). Since $(2, p)=1$, squaring map $x \rightarrow x^{2}$ on a $p$-group is an automorphism. Thus, -1 is a square in $R^{*}$ if and only if -1 is a square in $(R /(p))^{*}$. We note that $(R /(p))^{*}=\left(\mathbb{Z}_{p}[x] /(\bar{f})\right)^{*} \simeq \mathbb{F}_{p^{r}}^{*}$. Therefore, -1 is a square in $(R /(p))^{*} \Longleftrightarrow(-1)^{\frac{p^{r}-1}{2}}=1$ in $\mathbb{F}_{p^{r}}^{*}$, and this is equivalent to $p^{r}-1 \equiv 0(\bmod 4)$, so the assertion follows immediately.

The following lemma shows that when $p=2$, our building-up construction for self-dual codes over $\operatorname{GR}\left(2^{m}, r\right)$ works only for $m=1$, that is, over $\operatorname{GF}\left(2^{r}\right)$. In fact, constructions of self-dual codes over $\mathrm{GF}\left(2^{r}\right)$ are developed in [17, 19, 20].

Lemma 3.2. Let $p=2$. Then -1 is a square in $\operatorname{GR}\left(2^{m}, r\right)$ if and only if $m=1$.

Proof. If $m=1$ then it is clear that -1 is a square in $\operatorname{GR}(2, r)$.

For the case $m \geq 2$, let $\operatorname{GR}\left(2^{m}, r\right)=\mathbb{Z}_{2^{m}}[x] /(f)$ and $\phi: \mathbb{Z}_{2^{m}}[x] \rightarrow \mathbb{Z}_{2}[x]$ be the reduction mod 2 map. It remains to show that -1 is a non-square in $\operatorname{GR}\left(2^{m}, r\right)$ for $m \geq 2$. To get a contradiction, assume that there exists $c$ in $\mathbb{Z}_{2^{m}}[x] /(f)$ with $c^{2}+1=0$ in $\mathbb{Z}_{2^{m}}[x] /(f)$. Then $c$ must be a non-constant as there is no constant $c$ in $\mathbb{Z}_{2^{m}}$ with $c^{2}+1=0$ in $\mathbb{Z}_{2^{m}}$. By our assumption, there exists $\hat{c}(x) \in \mathbb{Z}_{2^{m}}[x]$ such that $\hat{c}(x)^{2}+1 \equiv 0(\bmod f)$, that is, $\hat{c}(x)^{2}+1=f g$ for some $g \in \mathbb{Z}_{2^{m}}[x]$, then we have $\phi(\hat{c})^{2}+1=\phi(f) \phi(g)$ in $\mathbb{Z}_{2}[x]$. As $f$ 
is monic basic irreducible, $\phi(f)$ is also irreducible polynomial in $\mathbb{Z}_{2}[x]$ of the same degree as $f$, so $\phi(f) \mid \phi(\hat{c})^{2}+1=(\phi(\hat{c})+1)^{2}$ in $\mathbb{Z}_{2}[x]$, which implies that $\phi(f) \mid \phi(\hat{c})+1$, i.e., $\phi(\hat{c})+1=\phi(f) \phi(h)$ for some polynomial $h \in \mathbb{Z}_{2^{m}}[x]$. Therefore, $\hat{c}+1-f h$ is in the kernel of $\phi$, so $\hat{c}+1=f h+2 s$ for some $s \in \mathbb{Z}_{2^{m}}[x]$; then $\hat{c}^{2}+1=f\left(f h^{2}+2 h(2 s-1)\right)+\left(4 s^{2}-4 s+2\right)$. Since $\hat{c}^{2}+1 \equiv 0(\bmod f), 2\left(2 s^{2}-2 s+1\right) \equiv 0(\bmod f)$, i.e., $2\left(2 s^{2}-2 s+1\right)=0$ in $\mathbb{Z}_{2^{m}}[x] /(f)$. As (2) is the maximal ideal of $\mathbb{Z}_{2^{m}}[x] /(f)$ and $2 s^{2}-2 s+1 \notin(2), 2 s^{2}-2 s+1$ is a unit of $\mathbb{Z}_{2^{m}}[x] /(f)$. Thus, $2\left(2 s^{2}-2 s+1\right)=0$ in $\mathbb{Z}_{2^{m}}[x] /(f)$ implies that $2=0$ in $\mathbb{Z}_{2^{m}}[x] /(f)$, a contradiction as $m \geq 2$. The result therefore follows.

Lemma 3.3. $(i)$ Let $\mathcal{C}$ be a self-orthogonal code over $\operatorname{GR}\left(p^{m}, r\right)$ of length $n$. Then $\mathcal{C}$ is self-dual if and only if $|\mathcal{C}|=\left|\mathrm{GR}\left(p^{m}, r\right)\right|^{\frac{n}{2}}=p^{\frac{m r n}{2}}$.

(ii) If there is $c \in \operatorname{GR}\left(p^{m}, r\right)$ such that $c^{2}=-1$ in $\operatorname{GR}\left(p^{m}, r\right)$, then there exist self-dual codes over $\operatorname{GR}\left(p^{m}, r\right)$ for all even lengths.

(iii) If $m$ is even, then there exist self-dual codes over $\operatorname{GR}\left(p^{m}, r\right)$ for all lengths.

(iv) If both $m$ and $r$ are odd and there is $c \in \operatorname{GR}\left(p^{m}, r\right)$ with $c^{2}=-1$, then there exist self-dual codes of length $n$ over $\operatorname{GR}\left(p^{m}, r\right)$ if and only if $n$ is even.

(v) If $m$ is odd and $p \equiv 3(\bmod 4)$, then there exist self-dual codes over $\operatorname{GR}\left(p^{m}, r\right)$ of all lengths a multiple of 4 .

Proof. (i) is obvious. For (ii), if there is $c \in \operatorname{GR}\left(p^{m}, r\right)$ such that $c^{2}=-1$ in $\operatorname{GR}\left(p^{m}, r\right)$, then $(1, c)$ generates a self-dual code of length 2 , therefore, there exist self-dual codes over $\operatorname{GR}\left(p^{m}, r\right)$ for all even lengths by the direct sum construction.

For (iii), assume that $m$ is even, then letting $\alpha=p^{\frac{m}{2}}$, the matrix $(\alpha)$ generates a selforthogonal code $\mathcal{C}$ of length 1 . Since $|\mathcal{C}|=p^{\frac{m r}{2}}$, the code $\mathcal{C}$ is a self-dual code of length 1 by $(i)$. Thus, there exist self-dual codes over $\operatorname{GR}\left(p^{m}, r\right)$ for all lengths.

To see $(i v)$, assume that there is a self-dual code $\mathcal{C}$ of odd length $n$ over $\operatorname{GR}\left(p^{m}, r\right)$, then $|\mathcal{C}|=p^{\frac{m r n}{2}}$. As $n$ is odd, $2 \mid m r$. If both $m$ and $r$ are odd, then there is no self-dual code over $\operatorname{GR}\left(p^{m}, r\right)$ of odd length $n$. Hence, if there exists a self-dual code over $\operatorname{GR}\left(p^{m}, r\right)$, then $n$ is even, and the other direction follows by $(i i)$.

For the proof of $(v)$, we refer to [14].

In the following proposition, we give the building-up construction for generating self-dual codes over $\operatorname{GR}\left(p^{m}, r\right)$.

Proposition 3.4. Assume that there exists $c$ in $\operatorname{GR}\left(p^{m}, r\right)$ such that $c^{2}=-1$ in $\operatorname{GR}\left(p^{m}, r\right)$. Let $G_{0}=\left(\mathbf{r}_{i}\right)$ be a generator matrix (not necessarily in standard form) of a self-dual code $\mathcal{C}_{0}$ over $\operatorname{GR}\left(p^{m}, r\right)$ of even length $n$, where $\mathbf{r}_{i}$ are the row vectors of the matrix $G_{0}$ respectively for $1 \leq i \leq e$. Let $\mathbf{x}=\left(x_{1}, \cdots, x_{j}, \cdots, x_{n}\right)$ be a vector in $\operatorname{GR}\left(p^{m}, r\right)^{n}$ with $\mathbf{x} \cdot \mathbf{x}=-1$ in 
$\operatorname{GR}\left(p^{m}, r\right)$. Suppose that $y_{i}:=\mathbf{x} \cdot\left(\mathbf{r}_{i}\right)$ for $1 \leq i \leq e$. Then the following matrix

$$
G=\left[\begin{array}{cc|ccccc}
1 & 0 & x_{1} & \cdots & x_{i} & \cdots & x_{n} \\
\hline-y_{1} & c y_{1} & & & & & \\
\vdots & \vdots & & & G_{0} & & \\
-y_{e} & c y_{e} & & & & &
\end{array}\right]
$$

generates a self-dual code $\mathcal{C}$ over $\operatorname{GR}\left(p^{m}, r\right)$ of length $n+2$.

Proof. We first show that any two rows of $G$ are orthogonal to each other. The first row of $G$ is orthogonal to itself as the inner product of the first row with itself equals $1+\mathbf{x} \cdot \mathbf{x}=0$ in $\operatorname{GR}\left(p^{m}, r\right)$. Furthermore, the first row of $G$ is orthogonal to any $(i+1)$ th row of $G$ for $1 \leq i \leq e-1$ since

$$
\begin{aligned}
& \left(1,0, x_{1}, \cdots, x_{i}, \cdots, x_{n}\right) \cdot\left(-y_{i}, c y_{i}, \mathbf{r}_{i}\right) \\
& =-y_{i}+\left(x_{1}, \cdots, x_{i}, \cdots, x_{n}\right) \cdot\left(\mathbf{r}_{i}\right)=0 .
\end{aligned}
$$

It now remains to show that any $(i+1)$ th row of $G$ is orthogonal to any $(j+1)$ th row for $1 \leq i, j \leq e-1$. The inner product of the $(i+1)$ th row of $G$ with the $(j+1)$ th row equals 0 since

$$
y_{i} y_{j}+c^{2} y_{i} y_{j}+\left(\mathbf{r}_{i}\right) \cdot\left(\mathbf{r}_{j}\right)=\left(1+c^{2}\right) y_{i} y_{j}=0 \quad \text { in } \operatorname{GR}\left(p^{m}, r\right) .
$$

Therefore, $\mathcal{C}$ is self-orthogonal, so $\mathcal{C} \subseteq \mathcal{C}^{\perp}$.

We note that $\left|\mathcal{C}_{0}\right|=p^{\frac{m r n}{2}}$ by $(i)$ of Lemma 3.3 as it is self-dual. Since $G$ had one more row starting with 1 than $G_{0}$, the code $\mathcal{C}$ generated by $G$ has $|\mathcal{C}|=p^{m r}\left|\mathcal{C}_{0}\right|=p^{\frac{m r(n+2)}{2}}$. This shows that $\mathcal{C}$ is self-dual by $(i)$ of Lemma 3.3. This completes the proof.

The following proposition shows that any self-dual code $\mathcal{C}$ over $\operatorname{GR}\left(p^{m}, r\right)$ with free rank $\geq 2$, even length $n \geq 4$ and minimum weight $d>2$ is obtained from some self-dual code $\mathcal{C}_{0}$ over $\operatorname{GR}\left(p^{m}, r\right)$ of length $n-2$ (up to permutation equivalence) by the construction method in Proposition 3.4.

Proposition 3.5. Assume that there exists $c$ in $\mathrm{GR}\left(p^{m}, r\right)$ such that $c^{2}=-1$ in $\operatorname{GR}\left(p^{m}, r\right)$. Any self-dual code $\mathcal{C}$ over $\mathrm{GR}\left(p^{m}, r\right)$ with the free rank $\geq 2$, even length $n \geq 4$ and minimum weight $d>2$ is obtained from some self-dual code $\mathcal{C}_{0}$ over $\operatorname{GR}\left(p^{m}, r\right)$ of length $n-2$ (up to permutation equivalence) by the construction method in Proposition 3.4.

Proof. Let $\mathcal{C}$ be a self-dual code over $\operatorname{GR}\left(p^{m}, r\right)$ of length $n$ with the free rank $\geq 2$ and minimum weight $d>2$. Let $G$ be a generator matrix of $\mathcal{C}$ in a standard form as follows:

$$
G=\left[\begin{array}{cc|c}
1 & 0 & \mathbf{a}_{1} \\
0 & 1 & \mathbf{a}_{2} \\
\hline 0 & 0 & \mathbf{a}_{3} \\
\vdots & \vdots & \vdots \\
0 & 0 & \mathbf{a}_{e}
\end{array}\right]
$$


where $\mathbf{a}_{i}$ are row vectors of length $n-2$. It is sufficient to show that there exist a vector $\mathbf{x}=\left(x_{1}, \cdots, x_{j}, \cdots, x_{n-2}\right)$ in $\operatorname{GR}\left(p^{m}, r\right)^{n-2}$ and a self-dual code $\mathcal{C}_{0}$ over $\operatorname{GR}\left(p^{m}, r\right)$ of length $n-2$ such that the code $\mathcal{C}_{1}$ constructed from $\mathcal{C}_{0}$ using $\mathbf{x}$ by the method in Proposition 3.4 is equivalent to $\mathcal{C}$.

We note that $\mathbf{a}_{i} \cdot \mathbf{a}_{j}=0$ for $i \neq j, 1 \leq i, j \leq e, \mathbf{a}_{i} \cdot \mathbf{a}_{i}=-1$ for $i=1,2$, and $\mathbf{a}_{i} \cdot \mathbf{a}_{i}=0$ for $3 \leq i \leq e$ since $\mathcal{C}$ is self-dual. Let $c$ be in $\operatorname{GR}\left(p^{m}, r\right)$ such that $c^{2}=-1$ in $\operatorname{GR}\left(p^{m}, r\right)$. We note that $c$ is a unit in $\operatorname{GR}\left(p^{m}, r\right)$, so $\mathcal{C}$ also has the following generator matrix

$$
G^{\prime}=\left[\begin{array}{cc|c}
1 & -c & \mathbf{a}_{1}-c \mathbf{a}_{2} \\
0 & -c & -c \mathbf{a}_{2} \\
\hline 0 & 0 & \mathbf{a}_{3} \\
\vdots & \vdots & \vdots \\
0 & 0 & \mathbf{a}_{e}
\end{array}\right] .
$$

Deleting the first two columns and the second row of $G^{\prime}$ produces an $(e-1) \times(n-2)$ matrix

$$
G_{0}:=\left[\begin{array}{c}
\mathbf{a}_{1}-c \mathbf{a}_{2} \\
\mathbf{a}_{3} \\
\vdots \\
\mathbf{a}_{e}
\end{array}\right] .
$$

It is straightforward to show that any two rows of $G_{0}$ are orthogonal to each other. Therefore, $G_{0}$ is a generator matrix of some self-orthogonal code $\mathcal{C}_{0}$ of length $n-2$.

Letting $\mathbf{x}=\mathbf{a}_{1}$ be a row vector of length $n-2, \mathbf{x} \cdot \mathbf{x}=\mathbf{a}_{1} \cdot \mathbf{a}_{1}=-1$ in $\operatorname{GR}\left(p^{m}, r\right)$. Using the vector $\mathbf{x}=\mathbf{a}_{1}$ and the self-orthogonal code $\mathcal{C}_{0}$, we can construct a self-orthogonal code $\mathcal{C}_{1}$ with the following generator matrix $G_{1}$ by Proposition 3.4. In fact, the $e \times n$ matrix

$$
G_{1}:=\left[\begin{array}{cc|c}
1 & 0 & \mathbf{a}_{1} \\
\hline 1 & -c & \mathbf{a}_{1}-c \mathbf{a}_{2} \\
0 & 0 & \mathbf{a}_{3} \\
\vdots & \vdots & \vdots \\
0 & 0 & \mathbf{a}_{e}
\end{array}\right]
$$

is permutation equivalent to $G$ as $c$ is a unit in $\operatorname{GR}\left(p^{m}, r\right)$, so, in fact, $\mathcal{C}_{1}$ is self-dual.

We therefore have that $\mathcal{C}_{0}$ is self-orthogonal and $|\mathcal{C}|=p^{\frac{m r n}{2}}$. Clearly $\left|\mathcal{C}_{0}\right|=|\mathcal{C}| / p^{m r}=$ $p^{\frac{m r(n-2)}{2}}$, thus $\mathcal{C}_{0}$ is also self-dual by $(i)$ of Lemma 3.3. Hence the given code $\mathcal{C}$ is obtained from the self-dual code $\mathcal{C}_{0}$ by the building-up construction in Proposition 3.4 (up to permutation equivalence). This completes the proof.

Remark 3.6. First, we note that a similar construction method can be applied to self-dual codes over finite chain rings. 
Second, we remark that the minimum weight of the code obtained by Proposition 3.4 can increase by up to 2 in general (although all examples in the next section have minimum weight increased by at most 1 ). It is affected by a coset leader of any coset of $\mathcal{C}_{0}$. Any two vectors $\mathbf{x}_{1}$ and $\mathbf{x}_{2}$ in the same coset of $\mathcal{C}_{0}$ produce the same code. Coset leaders of $\mathcal{C}_{0}$ with higher weights usually produce self-dual $\operatorname{codes} \mathcal{C}$ with better minimum weights. Finding their exact minimum weights can be time-consuming in this construction.

\section{Examples}

In this section, we construct MDS self-dual codes over various Galois rings using the building-up construction. Checking equivalence of codes over Galois rings seems difficult. Thus we generate usually 30 distinct (not necessarily inequivalent) codes and list 10 of them to save the space. Omitted codes in this section are available from the authors.

As our self-dual codes $\mathcal{C}$ will be free, we use the following lemma to expedite the calculation of the minimum weight of $\mathcal{C}$.

Lemma 4.1. ([26, Cor. 4.3]) If $\mathcal{C}$ is a free code over a finite chain ring $R$ (equivalently, a finite local principal ideal ring $R$ ), then $d(\mathcal{C})=d(\overline{\mathcal{C}})$, where $\overline{\mathcal{C}}=\{\bar{c} \mid c \in \mathcal{C}\}$ and $\bar{c}$ is the image of $c$ under the projection of $R^{n}$ onto $K^{n}$, extended coordinatewisely from the projection of $R$ to its residue field $K$.

Example 4.2. Consider $\operatorname{GR}\left(3^{2}, 2\right)$. We regard this as $\mathbb{Z}_{3^{2}}[w]=\left\{a+b w \mid a, b \in \mathbb{Z}_{3^{2}}\right\}$, where $w$ satisfies $w^{2}=7 w+7$. Then $-1=c^{2}$ for some $c$ in $\operatorname{GF}\left(3^{2}, 2\right)$ by Lemma 3.1. Take $c=w+1$ as $c^{2}=-1$. The generator matrix $\left[\begin{array}{ll}1 & c\end{array}\right]$ is a self-dual code $\mathcal{C}_{2}$ of length 2 over $\operatorname{GR}\left(3^{2}, 2\right)$ by Proposition 3.4, whose Hamming weight enumerator is $W_{2}(y)=1+80 y^{2}$. We have found at least 30 MDS self-dual codes of length 4, all of whose Hamming weight enumerators are $W_{4}(y)=1+320 y^{3}+6240 y^{4}$. For example, $\{(1,0,1,4),(5 w+4, w+6,1, w+1)\}$ generates a self-dual code $\mathcal{C}_{4}$ of length 4 with Hamming weight 3 . We list only 10 distinct codes in Table 2 to save space. Here by Proposition 3.4, the second and third columns give the values of $-y_{1}$ and $c y_{1}$, respectively, and the fourth column gives the value of $\mathbf{x}$.

Applying Proposition 3.4 to $\mathcal{C}_{4}$ we obtain at least 30 MDS self-dual codes of length 6 , all of whose Hamming weight enumerators are $W_{6}(y)=1+1200 y^{4}+36960 y^{5}+493280 y^{6}$. For example, $\{(1,0,0,1, w+1, w+1),(4 w+4,4,1,0,1,4),(7 w+3,4 w+2,5 w+4, w+6,1, w+1)\}$ generates a self-dual code $\mathcal{C}_{6}$ of length 6 with Hamming weight 4 . We list only 10 distinct codes in Table 3 to save space. Here columns from the second to sixth give the values of $-y_{1}, c y_{1},-y_{2}, c y_{2}$, respectively, and the last column gives the value of $\mathbf{x}$.

Similarly, using $\mathcal{C}_{6}$, we obtain at least 30 MDS self-dual codes of length 8 , all of whose Hamming weight enumerators are $W_{8}(y)=1+4480 y^{5}+170240 y^{6}+3897600 y^{7}+38974400 y^{8}$. One example, denoted by $\mathcal{C}_{8}$, is generated by $\{(1,0,0,0,1, w, 2,2 w+7),(w+4,6 w+7,1,0,0,1, w+$ $1, w+1),(w+5,5 w+6,4 w+4,4,1,0,1,4),(4 w+2,2 w+6,7 w+3,4 w+2,5 w+4, w+6,1, w+1)\}$. 
Table 2: MDS self-dual codes of length 4 over $\operatorname{GR}\left(3^{2}, 2\right)$ from $\mathcal{C}_{2}$

\begin{tabular}{|c|c|c|c|}
\hline codes & $-y_{1}$ & $c y_{1}$ & $\mathbf{x}$ \\
\hline $\mathcal{C}_{4,1}$ & $4 w+3$ & $w+5$ & $(1,5)$ \\
$\mathcal{C}_{4,2}$ & $7 w+5$ & $2 w$ & $(2,2)$ \\
$\mathcal{C}_{4,3}$ & $2 w$ & $2 w+4$ & $(2,7)$ \\
$\mathcal{C}_{4,4}$ & $8 w+4$ & $4 w+3$ & $(4,1)$ \\
$\mathcal{C}_{4,5}$ & $w+6$ & $4 w+5$ & $(4,8)$ \\
$\mathcal{C}_{4,6}$ & $8 w+3$ & $5 w+4$ & $(5,1)$ \\
$\mathcal{C}_{4,7}$ & $w+5$ & $5 w+6$ & $(5,8)$ \\
$\mathcal{C}_{4,8}$ & $7 w$ & $7 w+5$ & $(7,2)$ \\
$\mathcal{C}_{4,9}$ & $2 w+4$ & $7 w$ & $(7,7)$ \\
$\mathcal{C}_{4,10}$ & $5 w+6$ & $8 w+4$ & $(8,4)$ \\
\hline
\end{tabular}

We list only 10 distinct codes in Table 4 to save space. Here columns from the second to sixth give the values of $-y_{1},-y_{2},-y_{3}$, respectively, from which $c y_{i}(i=1,2,3)$ is easily computed, and the last column gives the value of $\mathbf{x}$.

For length 10 , using $\mathcal{C}_{8}$, we obtain at least 30 self-dual codes of length 10 with Hamming minimum distance 5 . We obtain at least 6 different Hamming weight enumerators as follows. $W_{10,1}(y)=1+288 y^{5}+15360 y^{6}+722880 y^{7}+21651120 y^{8}+384933440 y^{9}+\cdots, W_{10,2}(y)=$ $1+432 y^{5}+14640 y^{6}+724320 y^{7}+21649680 y^{8}+384934160 y^{9}+\cdots, W_{10,3}(y)=1+720 y^{5}+$ $13200 y^{6}+727200 y^{7}+21646800 y^{8}+384935600 y^{9}+\cdots, W_{10,4}(y)=1+576 y^{5}+13920 y^{6}+$ $725760 y^{7}+21648240 y^{8}+384934880 y^{9}+\cdots, W_{10,5}(y)=1+144 y^{5}+16080 y^{6}+721440 y^{7}+$ $21652560 y^{8}+384932720 y^{9}+\cdots$, or $W_{10,6}(y)=1+128 y^{5}+16160 y^{6}+721280 y^{7}+21652720 y^{8}+$ $384932640 y^{9}+\cdots$.

We list them in Table 5, where the last column displays the corresponding weight enumerator. 
Table 3: MDS self-dual codes of length 6 over $\operatorname{GR}\left(3^{2}, 2\right)$ from $\mathcal{C}_{4}$

\begin{tabular}{|c|c|c|c|c|c|}
\hline codes & $-y_{1}$ & $c y_{1}$ & $-y_{2}$ & $c y_{2}$ & $\mathbf{x}$ \\
\hline $\mathcal{C}_{6,1}$ & $4 w+4$ & 4 & $w+3$ & $7 w+8$ & $(0,1, w+4, w+7)$ \\
$\mathcal{C}_{6,2}$ & $4 w+4$ & 4 & $4 w+3$ & $w+5$ & $(0,1, w+7, w+4)$ \\
$\mathcal{C}_{6,3}$ & $5 w+5$ & 5 & $6 w+6$ & 6 & $(0,1,2 w+2,5 w+5)$ \\
$\mathcal{C}_{6,4}$ & $5 w+5$ & 5 & 6 & $3 w+3$ & $(0,1,2 w+5,5 w+2)$ \\
$\mathcal{C}_{6,5}$ & $5 w+5$ & 5 & $3 w+6$ & $6 w$ & $(0,1,2 w+8,5 w+8)$ \\
$\mathcal{C}_{6,6}$ & $4 w+4$ & 4 & $w+6$ & $4 w+5$ & $(0,1,4 w+1,7 w+1)$ \\
$\mathcal{C}_{6,7}$ & $4 w+4$ & 4 & $4 w+6$ & $7 w+2$ & $(0,1,4 w+4,7 w+7)$ \\
$\mathcal{C}_{6,8}$ & $4 w+4$ & 4 & $7 w+6$ & $w+8$ & $(0,1,4 w+7,7 w+4)$ \\
$\mathcal{C}_{6,9}$ & $5 w+5$ & 5 & 0 & 0 & $(0,1,5 w+2,2 w+5)$ \\
$\mathcal{C}_{6,10}$ & $5 w+5$ & 5 & $3 w$ & $3 w+6$ & $(0,1,5 w+5,2 w+2)$ \\
\hline
\end{tabular}

Table 4: MDS self-dual codes of length 8 over $\operatorname{GR}\left(3^{2}, 2\right)$ from $\mathcal{C}_{6}$

\begin{tabular}{|c|c|c|c|c|}
\hline codes & $-y_{1}$ & $-y_{2}$ & $-y_{3}$ & \multicolumn{1}{|c|}{$\mathbf{x}$} \\
\hline $\mathcal{C}_{8,1}$ & $4 w+4$ & $4 w+2$ & $w+2$ & $(0,0,1, w, 5,8 w+7)$ \\
$\mathcal{C}_{8,2}$ & $7 w+4$ & $7 w+8$ & $7 w+2$ & $(0,0,1, w, 8,5 w+7)$ \\
$\mathcal{C}_{8,3}$ & $4 w+7$ & $w+8$ & $w+8$ & $(0,0,1, w, 3 w+2,8 w+4)$ \\
$\mathcal{C}_{8,4}$ & $7 w+7$ & $4 w+5$ & $7 w+8$ & $(0,0,1, w, 3 w+5,5 w+4)$ \\
$\mathcal{C}_{8,5}$ & $w+7$ & $7 w+2$ & $4 w+8$ & $(0,0,1, w, 3 w+8,2 w+4)$ \\
$\mathcal{C}_{8,6}$ & $7 w+1$ & $w+2$ & $7 w+5$ & $(0,0,1, w, 6 w+2,5 w+1)$ \\
$\mathcal{C}_{8,7}$ & $w+1$ & $4 w+8$ & $4 w+5$ & $(0,0,1, w, 6 w+5,2 w+1)$ \\
$\mathcal{C}_{8,8}$ & $4 w+1$ & $7 w+5$ & $w+5$ & $(0,0,1, w, 6 w+8,8 w+1)$ \\
$\mathcal{C}_{8,9}$ & $4 w+4$ & $8 w+6$ & $w+8$ & $(0,0,1, w+2, w, 5)$ \\
$\mathcal{C}_{8,10}$ & $4 w+7$ & $5 w+3$ & $4 w+2$ & $(0,0,1, w+2, w+3,3 w+5)$ \\
\hline
\end{tabular}

Table 5: Self-dual codes of length 10 with $d_{H}=5$ over $\operatorname{GR}\left(3^{2}, 2\right)$ from $\mathcal{C}_{8}$

\begin{tabular}{|c|c|c|c|c|c|c|}
\hline codes & $-y_{1}$ & $-y_{2}$ & $-y_{3}$ & $-y_{4}$ & $\mathbf{x}$ & Wt. En. \\
\hline $\mathcal{C}_{10,1}$ & $6 w+6$ & $6 w+5$ & 7 & $7 w+3$ & $(0,0,0,1,1,1,2,1)$ & $W_{10,1}$ \\
$\mathcal{C}_{10,2}$ & $w+2$ & $8 w+7$ & 6 & 5 & $(0,0,0,1,1,1,2,8)$ & $W_{10,1}$ \\
$\mathcal{C}_{10,3}$ & 6 & 8 & 1 & $4 w+6$ & $(0,0,0,1,1,1,5,4)$ & $W_{10,2}$ \\
$\mathcal{C}_{10,4}$ & $3 w$ & $6 w+2$ & $3 w+1$ & $4 w+3$ & $(0,0,0,1,1,1,3 w+5,3 w+4)$ & $W_{10,2}$ \\
$\mathcal{C}_{10,5}$ & 0 & $3 w+8$ & $3 w+7$ & $7 w$ & $(0,0,0,1,1,1,3 w+2,3 w+1)$ & $W_{10,3}$ \\
$\mathcal{C}_{10,6}$ & $7 w+5$ & $8 w+7$ & 6 & $6 w+2$ & $(0,0,0,1,1,1,6 w+2,3 w+8)$ & $W_{10,4}$ \\
$\mathcal{C}_{10,7}$ & $3 w+3$ & 2 & $6 w+7$ & $7 w+6$ & $(0,0,0,1,1,1,6 w+2,6 w+1)$ & $W_{10,4}$ \\
$\mathcal{C}_{10,8}$ & $5 w+8$ & $6 w+8$ & $2 w+3$ & $7 w+1$ & $(0,0,0,1,1,2,3,4 w+4)$ & $W_{10,5}$ \\
$\mathcal{C}_{10,9}$ & $3 w+2$ & $2 w$ & 0 & $5 w+2$ & $(0,0,0,1,1,2,5,2)$ & $W_{10,5}$ \\
$\mathcal{C}_{10,10}$ & 4 & $6 w$ & $4 w+5$ & $8 w+1$ & $(0,0,0,1,1,2,5 w+5,3)$ & $W_{10,6}$ \\
\hline
\end{tabular}


In the following examples, using the building-up construction in Proposition 3.4, we obtain self-dual (near) MDS codes over $\operatorname{GR}(q, 2)$ with $q$ odd, $25 \leq q \leq 125$ and lengths up to 12 .

Example 4.3. Consider $\operatorname{GR}\left(5^{2}, 2\right)$. We regard this as $\mathbb{Z}_{5^{2}}[w]=\left\{a+b w \mid a, b \in \mathbb{Z}_{5^{2}}\right\}$, where $w$ satisfies $w^{2}=21 w+23$. Then $-1=c^{2}$ for some $c$ in $\operatorname{GF}\left(5^{2}, 2\right)$ by Lemma 3.1, hence we take $c=7$ as $c^{2}=-1$.

We give the generator matrix $G_{1}$ in the appendix for a self-dual near-MDS $[12,6,6]$ code over $\operatorname{GR}\left(5^{2}, 2\right)$. It has the property that the successive deletion of the first two columns and the first row of $G_{1}$ produces MDS self-dual codes of lengths 10,8,6,4, and 2. We have found at least 30 self-dual near-MDS $[12,6,6]$ codes whose successive deletion as above produces MDS self-dual codes of lengths 10, 8, 6, 4, and 2.

Example 4.4. Consider $\operatorname{GR}\left(3^{3}, 2\right)$. We regard this as $\mathbb{Z}_{3^{3}}[w]=\left\{a+b w \mid a, b \in \mathbb{Z}_{3^{3}}\right\}$, where $w$ satisfies $w^{2}=25 w+25$. Then $-1=c^{2}$ for some $c$ in $\operatorname{GF}\left(3^{3}, 2\right)$ by Lemma 3.1, so we take $c=w+1$ as $c^{2}=-1$.

We give the following generator matrix $G_{2}$ for a self-dual near-MDS $[10,5,5]$ code over $\operatorname{GR}\left(3^{3}, 2\right)$.

$$
G_{2}:=\left[\begin{array}{cccccccccc}
1 & 0 & 15 w+26 & 10 w+5 & 16 w+22 & 3 w+23 & 7 w+9 & 4 w+12 & 25 w+17 & 9 w+14 \\
16 w & 16 w+5 & 1 & 0 & 18 w+16 & 23 w+8 & 5 w+17 & w+7 & 6 w & 19 \\
16 w+15 & w+17 & 6 w+8 & 25 w+4 & 1 & 0 & 0 & 1 & w+1 & 26 w+26 \\
20 w+26 & 21 w+14 & 13 w+17 & 23 w+9 & 4 w+4 & 4 & 1 & 0 & 1 & 5 \\
6 w+12 & 21 w & 3 w+12 & 18 w+21 & 25 w+2 & 23 w+21 & 22 w+21 & w+23 & 1 & w+1
\end{array}\right]
$$

Just like Example 4.3, $G_{2}$ produces MDS self-dual codes of lengths 8,6,4, and 2. We have found at least 30 self-dual near-MDS $[10,5,5]$ codes having this property.

Example 4.5. Consider $\operatorname{GR}\left(7^{2}, 2\right)$. We regard this as $\mathbb{Z}_{7^{2}}[w]=\left\{a+b w \mid a, b \in \mathbb{Z}_{7^{2}}\right\}$, where $w$ satisfies $w^{2}=43 w+46$. Then $-1=c^{2}$ for some $c$ in $\operatorname{GF}\left(7^{2}, 2\right)$ by Lemma 3.1, so we take $c=20 w+11$ as $c^{2}=-1$.

A generator matrix $G_{3}$ for a self-dual near-MDS $[12,6,6]$ code over $\operatorname{GR}\left(7^{2}, 2\right)$ is given in the appendix. As above, $G_{3}$ produces MDS self-dual codes of lengths $10,8,6,4$, and 2 . We have found at least 30 self-dual near-MDS $[12,6,6]$ codes having this property.

Example 4.6. Consider $\operatorname{GR}\left(3^{4}, 2\right)$. We regard this as $\mathbb{Z}_{3^{4}}[w]=\left\{a+b w \mid a, b \in \mathbb{Z}_{3^{4}}\right\}$, where $w$ satisfies $w^{2}=79 w+79$. Then $-1=c^{2}$ for some $c$ in $\operatorname{GF}\left(3^{4}, 2\right)$ by Lemma 3.1, so we take $c=w+1$ as $c^{2}=-1$.

We give the following generator matrix $G_{4}$ for a self-dual near-MDS $[10,5,5]$ code over $\operatorname{GR}\left(3^{4}, 2\right)$.

$$
G_{4}:=\left[\begin{array}{cccccccccc}
1 & 0 & 19 w+35 & 19 w+15 & 39 w+2 & 3 w+54 & 21 w+15 & 30 w+46 & 79 w+25 & 12 w+8 \\
51 w+25 & 26 w+77 & 1 & 0 & 69 w+75 & 57 w+56 & 47 w+73 & 37 w+64 & 55 w+70 & 38 w+6 \\
23 w+38 & 66 w+8 & 73 w+52 & 21 w+13 & 1 & 0 & 0 & 1 & w+1 & w+1 \\
77 w+39 & 38 w+34 & 25 w+50 & 56 w & 58 w+58 & 58 & 1 & 0 & 1 & 22 \\
47 w+14 & 33 w+80 & 61 w+62 & 80 w+60 & 79 w+21 & 58 w+56 & 59 w+58 & w+60 & 1 & w+1
\end{array}\right]
$$


Then $G_{4}$ produces MDS self-dual codes of lengths 8,6,4, and 2 as before. We have found at least 30 self-dual near-MDS $[10,5,5]$ codes having this property.

Example 4.7. Consider $\operatorname{GR}\left(11^{2}, 2\right)$. We regard this as $\mathbb{Z}_{11^{2}}[w]=\left\{a+b w \mid a, b \in \mathbb{Z}_{11^{2}}\right\}$, where $w$ satisfies $w^{2}=114 w+119$. Then $-1=c^{2}$ for some $c$ in $\operatorname{GF}\left(11^{2}, 2\right)$ by Lemma 3.1, so we take $c=29 w+41$ as $c^{2}=-1$.

A generator matrix $G_{5}$ for a MDS self-dual $[12,6,7]$ code over $\operatorname{GR}\left(11^{2}, 2\right)$ is given in the appendix. Then $G_{5}$ produces MDS self-dual codes of lengths 10,8,6,4, and 2, as before. We have not found other MDS self-dual $[12,6,7]$ codes, but have found at least 30 self-dual near-MDS $[12,6,6]$ codes giving MDS self-dual codes of length 10,8,6, 4, and 2 .

Example 4.8. Consider $\operatorname{GR}\left(5^{3}, 2\right)$. We regard this as $\mathbb{Z}_{5^{3}}[w]=\left\{a+b w \mid a, b \in \mathbb{Z}_{5^{3}}\right\}$, where $w$ satisfies $w^{2}=121 w+123$. Then $-1=c^{2}$ for some $c$ in $\operatorname{GF}\left(5^{3}, 2\right)$ by Lemma 3.1, thus we take $c=57$ as $c^{2}=-1$.

We give a generator matrix $G_{6}$ in the appendix for a self-dual near-MDS $[12,6,6]$ code over $\operatorname{GR}\left(5^{3}, 2\right)$, whose successive deletion as before produces MDS self-dual codes of lengths $10,8,6,4$, and 2 . We have found at least such 30 self-dual near-MDS $[12,6,6]$ codes.

\section{ACKNOWLEDGMENT}

The authors thank the anonymous referees for comments and suggestions which helped to improve the paper.

\section{References}

[1] E. Bannai, S.T. Dougherty, M. Harada, and M. Oura, Type II codes, even unimodular lattices and invariant rings, IEEE Trans. Inform. Theory, Vol. 45, No. 4 (1999) pp. 1194-1205.

[2] I. F. Blake, Codes over certain rings, Inform. Contr., Vol. 20 (1972) pp. 396-404.

[3] I. F. Blake, Codes over integer residue rings, Inform. Contr., Vol. 29 (1975) pp. 295-300.

[4] A. Bonnecaze, P. Solé, C. Bachoc, B. Mourrain, Type II codes over $Z_{4}$, IEEE Trans. Inform. Theory., Vol. 43, No. 3 (1997) pp. 969-976.

[5] R.A Brualdi and V.S. Pless, Weight Enumerators of Self-Dual Codes, IEEE Trans. Inform. Theory, Vol. 37, No. 4 (1991) pp. 1222-1225.

[6] A.R. Calderbank and N.J.A. Sloane, Modular and p-adic cyclic codes, Des. Codes Cryptogr. Vol. 6, No. 1 (1995) pp. 21-35. 
[7] J. Cannon and C. Playoust, An Introduction to Magma, University of Sydney, Sydney, Australia, (1994).

[8] J.H. Conway, N.J.A. Sloane, Self-dual codes over the integers modulo 4, J. Combin. Theory Ser. A , Vol. 62, No. 1 (1993) pp. 30-45.

[9] S.T Dougherty, Shadow codes and weight enumerators, IEEE Trans. Inform. Theory, Vol. 41, No.3 (1995) pp. 762-768.

[10] S.T. Dougherty, T.A. Gulliver, and M. Harada, Type II self-dual codes over finite rings and even unimodular lattices, J. Algebraic Combin. Vol. 9, No. 3 (1999) pp. 233-250.

[11] S.T. Dougherty, T.A. Gulliver, Y.H. Park, J.N.C. Wong, Optimal linear codes over $\mathbb{Z}_{m}$, to appear in J. Korean Math. Soc.

[12] S.T. Dougherty, T.A. Gulliver, and J. Wang, Self-dual codes over $Z_{8}$ and $Z_{9}$, Designs, Codes and Cryptography, Vol. 41 (2006) pp. 235-249.

[13] S.T. Dougherty, M. Harada, P. Solé, Self-Dual Codes over Rings and the Chinese Remainder Theorem, Hokkaido Math Journal, Vol. 28 (1999) pp. 253-283.

[14] S.T. Dougherty, J.-L. Kim, and H. Kulosman, MDS codes over finite principal ideal rings, preprint.

[15] S.T. Dougherty and K. Shiromoto, MDR Codes over $\mathbb{Z}_{k}$, IEEE-IT, Vo.l 46, No. 1 (2000) 265-269.

[16] P. Gaborit, An. M. Natividad, and P. Solé, Eisenstein lattices, Galois rings and quaternary codes, International Journal of Number Theory, Vol. 2, No. 2 (2006) pp. 289-303.

[17] T.A. Gulliver, J.-L. Kim and Y. Lee, New MDS and Near-MDS Self-Dual Codes, preprint.

[18] A. R. Hammons, P.V. Kumar, A.R. Calderbank, N. J. A. Sloane, and P. Solé, The $\mathbb{Z}_{4}$-linearity of Kerdock, Preparata, Goethals, and related codes, IEEE Trans. Inform. Theory, Vol. 40, No. 2 (1994) pp. 301-319.

[19] J.-L. Kim, New self-dual codes over GF(4) with the highest known minimum weights, IEEE Trans. Inform. Theory, Vol. 47 (2001) pp. 1575-1580.

[20] J.-L. Kim and Y. Lee, Euclidean and Hermitian Self-Dual MDS Codes over Large Finite Fields, J. Combin. Theory Ser. A, Vol. 105 (2004) pp. 79-95.

[21] H. Lee and Y. Lee, Construction of self-dual codes over finite rings $\mathbb{Z}_{p^{m}}$, to appear in J. Combin. Theory Ser. A.

[22] B.R. McDonald, Finite Rings with identity, Pure and Applied Mathematics, Vol. 28. Marcel Dekker, Inc., New York, (1974). 
[23] F.J. MacWilliams and N.J.A. Sloane, The Theory of Error-Correcting Codes, Amsterdam, The Netherlands: North-Holland, (1977).

[24] G. Nebe, E. M. Rains and N. J. A. Sloane, Self-Dual Codes and Invariant Theory, Springer, Berlin, Feb. (2006).

[25] G.H. Norton and A. Salagean, On the structure of linear and cyclic codes over a finite chain ring, Appl. Algebra Engrg. Comm. Comput., Vol. 10, No. 6 (2000) pp. 489-506.

[26] G.H. Norton and A. Salagean, On the Hamming distance of linear codes over a finite chain ring, IEEE Trans. Inform. Theory, Vol. 46, No. 3 (2000) pp. 1060-1067.

[27] V. Pless, P. Solé, Z. Qian, Cyclic self-dual $\mathbb{Z}_{4}$-codes, Finite Fields Appl., Vol. 3, No. 1 (1997) pp. 48-69.

[28] E. Rains and N.J.A. Sloane, Self-dual codes, in Handbook of Coding Theory, V.S. Pless and W.C. Huffman, Eds. Amsterdam. The Netherlands: Elsevier, (1998).

[29] P. Shankar, On BCH codes over arbitrary integer rings, IEEE Trans. Inform. Theory, Vol. 25 (1979) pp. 480-483.

[30] E. Spiegel, Codes over $Z_{m}$, Inform. Contr., Vol. 35 (1977) pp. 48-52.

[31] E. Spiegel, Codes over $Z_{m}$ revisited, Inform. Contr., Vol. 37 (1978) pp. 100-104.

[32] Z.-X. Wan, Lectures on finite fields and Galois rings, World Scientific Publishing Co., Inc., River Edge, NJ, (2003).

[33] J.A. Wood, Duality for modules over finite rings and applications to coding theory, Amer. J. Math., Vol. 121, No. 3 (1999) pp. 555-575.

\section{Appendix}

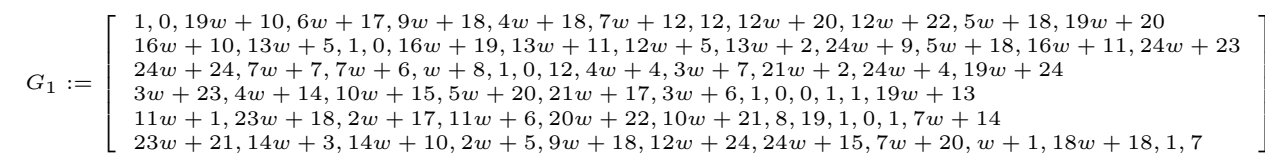

$G_{3}:=\left[\begin{array}{l}1,0,22 w+46,27 w+8,13 w+3,47 w+9,12 w+37,2 w+16,46 w+35,36 w+23,31 w+40,9 w+40 \\ 40 w+46,10 w+32,1,0,19 w+19,4 w+33,33 w+20,33 w+19,23 w+35,17 w+20,36 w+1,4 w+5 \\ 4 w+39,48 w+7,21 w+16,9 w+6,1,0,42 w+35,7 w+37,44 w+35,44 w+4,23 w+7,7 w+20 \\ 35,35 w+7,39 w+13,22 w+41,3 w+8,20 w+43,1,0,0,1,1,12 \\ 36 w+6,31 w+36,48 w+33,15 w+18,2 w+48,42 w+33, w+2,20 w+38,1,0,1,4 w+12 \\ 16 w+17,32 w+38,31 w+12,3 w+13,48 w+11,14 w+15,38 w+15,20 w+8,9,16 w+48,1,20 w+11\end{array}\right]$

$G_{5}:=\left[\begin{array}{l}1,0,24 w+4,14 w+25,103 w+94,61 w+6,58 w+12,100 w+76,67 w+90,98 w+20,68 w+113,42 w+36 \\ 4 w+32,83 w+9,1,0,2 w+5,81 w+34,13 w+16,60 w+106,97 w+67,85 w+55,21 w+110,21 w+75 \\ 11 w+73,28 w+65,72 w+115,101 w+66,1,0,85 w+38,107 w+85,80 w+5,66 w+25,2 w+51,98 w+76 \\ 79 w+20,118 w+11,54 w+73,97 w+18,62 w+10,74 w+40,1,0,0,1,1,57 w+18 \\ 45 w+82,72 w+95,93 w+58,74 w+112,4 w+74,75 w+102,6 w+20,29 w+12,1,0,1,19 \\ 67 w+59,68 w+15,106 w+25,112 w+41,107 w+35,105 w+52,92 w+4,26 w+90,54 w+67,29 w+22,1,29 w+41\end{array}\right]$ 
$[1,0,15 w+120,63 w+26,66 w+104,82 w+92,94 w+85,5 w+25,75 w+68,38 w+17,94 w+83,48 w+48$ $34 w+72,62 w+21,1,0,27 w+84,50 w+73,105 w+1,66 w+100,9 w+53,7 w+70,72 w+71,113 w+68$ $45 w+95,60 w+85,99 w+99,107 w+107,1,0,54 w+93,13 w+104,16 w+86,65 w+93,70 w+22,112 w+5$ $49 w+78,82 w+54,97 w+72,96 w+21,72 w+83,21 w+19,1,0,0,1,1,44 w+88$

$102 w+84,61 w+87,96 w+85,28 w+30,93 w+20,74 w+110,108,94,1,0,1,57 w+114$

$105 w+30,15 w+40,25 w+115,75 w+70,62 w+104,91 w+72,49 w+40,82 w+95, w+1,68 w+68,1,57$ 